$w x u$ and $y z u$ then the joining line of the two remaining points of intersection of the sextics so determined will meet $F$ elsewhere in the two lines $w x$ and $y z$.

CoRnelL UNIVERSITY, February, 1900 .

\title{
NO'TE ON THE GROUP OF ISOMORPHISMS.
}

\author{
BY DR. G. A. MILLER.
}

(Read before the American Mathematical Society, February 24, 1900.)

LET $s_{1}, s_{2}, \cdots, s_{g}$ represent all the operators of a group $G$ and let $t_{a} s_{a}$ correspond to $s_{a}(\alpha=1,2, \cdots, g)$ in any given simple isomorphism of $G$ with itself. It is evident that $t_{a}$ is some operator of $G$. When $G$ is abelian these $t_{a}$ 's must constitute a group $T$ which is isomorphic with $G$.* In this isomorphism, $\mathrm{t}_{\alpha}$ evidently can not be the inverse of $s_{a}$ unless $s_{a}=1$. As this condition is sufficient as well as necessary, we have

Theorem I.-Every simple isomorphism of an abelian group $A$ with itself may be obtained by $1^{\circ}$ making $A$ isomorphic with one of its subgroups or with itself in such a manner that no operator corresponds to its inverse, and $2^{\circ}$ making each operator of $A$ correspond to itself multiplied by the operator which corresponds to it in the given isomorphism.

The simplest case that can present itself is the one in which the subgroup of $G$, which corresponds to identity of $T$ in the given isomorphism between $G$ and $T$, includes $T$. The resulting simple isomorphism of $G$ with itself must correspond to an operator in the group of isomorphisms of $G$, whose order is equal to the operator of highest order in $T$. When the order of $T$ is an odd prime number $p$, or the double of an odd prime, only one other case can present itself ; viz, the case in which $T$ corresponds to itself, or to its subgroup of an odd prime order, in the given isomorphism between $G$ and $T$. The resulting simple isomorphism of $G$ with itself may clearly correspond to a cyclical group of order $p-1$, or to any one of its subgroups in the group of isomorphisms of $G$. These results lead to the following

\footnotetext{
* When $G$ is non-abelian, these $t a$ 's need not constitute a group, as can be seen from the simple isomorphisms of the symmetric group of order 6 with itself.
} 
Theorem II.-If we make an abelian group A simply isomorphic with itself by multiplying its operators by the operators of a subgroup whose order is an odd prime number $p$ or the double of this prime number, the resulting simple isomorphism of $A$ with itself will correspond to an operator of order $p, 2 p$, or $\frac{p-1}{\alpha}$ (a being any division of $p-1$ ) in the group of isomorphisms of $A$.*

The determination of all the possible orders of the corresponding operators in the group of isomorphisms of any abelian group, when $T$ is a given subgroup, seems to be a problem of considerable difficulty. When the order of $T$ is small the number of cases that have to be considered is also small. In addition to the orders included in the theorems given, we have the following when the order of $T$ does not exceed $8:$ If $T$ is the cyclical group of order four, the resulting isomorphism may correspond to an operator of order two in the group of isomorphisms, and when $T$ is the noncyclical group of this order, it may correspond to operators of orders 3 and 4 . When $T$ is the cyclical group of order 8 , the orders of these operators may be 2,4 , and 8 ; when $T$ is the direct product of the cyclical group of order 4 and an operator of order 2 , the orders of the corresponding operators in the group of isomorphisms may be 2 and 4 ; finally, when $T$ is the direct product of three operators of order 2 , the given operators may be of orders $2,3,4,6$, and 7 . While all of the possible cases for a given $T$ may present themselves in the same group it is evident that this does not always happen.

For the sake of illustration we may consider the group of isomorphisms of the group of order 8 which is the direct product of three operators of order 2. $\dagger$ Each of its 7 subgroups of order 4 leads to three operators of order two, according to the second sentence below Theorem I. We thus obtain the 21 operators of order 2 of the required group of isomorphisms when we consider all the possible instances in which the order of $T$ is 2 . If the order of $T$ is 4 , two cases present themselves-in one case just two of the operators of $T$ (including identity) correspond to operators of $T$, and in the other case each one of the operators of $T$ corresponds to some operator of $T$. The former case leads to the 42 operators of order 4 and the latter to 56 operators of order 3 of the required group of isomorphisms. Finally, we obtain 48 operators of order 7 when we consider all the pos-

* Cf. Comptes rendus, vol. 130, 1900, p. 316.

† Cf. Moore, Bulletin, vol. 1, 1894, p. 63. 
sible instances in which the order of $T$ is 8 . Hence we observe that the required group is the well known simple group of order 168.

It is known that there are abelian groups which cannot be groups of cogredient isomorphisms.* Suppose that the group of cogredient isomorphisms $H$ of a given group $G$ is Hamiltonian. Each of the operators of $G$ which corresponds to identity of $H$ is self-conjugate in $G$. Since the non-identity commutator of $H$ is some power of each one of a set of generators of $H$, all the operators of $G$ which correspond to it must also be self-conjugate in $G$. As this is clearly impossible, it follows that a Hamiltonian group cannot be a group of cogredient isomorphisms. In fact, the preceding arguments apply to all groups which contain an operator different from identity, which is some power of each one of a set of generating operators. Hence such a group cannot be the group of cogredient isomorphisms of any group whatsoever.

CORNELL UNIVERSITY, February, 1900.

\section{LOBACHEVSKY'S GEOMETRY.}

Nikolaj Iwanowitsch Lobatschefskij. Zwei Geometrische Abhandlungen aus dem Russischen übersetzt, mit Anmerkungen und mit einer Biographie des Verfassers von FRIEDRICH Engel. Leipzig, B. G. Teubner, 1899. 8vo. xvi +476 pp.

This volume is the first in the series of the "Urkunden zur Geschichte der Nichteuklidischen Geometrie" planned by Friedrich Engel and Paul Stäckel in continuation of their "Theorie der Parallellinien von Euklid bis auf Gauss." The present work on Lobachevsky has been undertaken by Engel, while a volume on the two Bolyais by Stäckel is soon to appear. All geometricians will welcome the opportunity thus afforded of becoming fully acquainted with the writings of these men, who share with Gauss the honor of being the first to break loose from the authority of Euclid and to show that the latter's axiom concerning parallel lines is not only incapable of proof but entirely unnecessary in a logical geometry which fully explains all facts of experience. The earlier works of Lobachevsky have been until now locked up in the original Russian and their contents

*Comptes rendus, vol. 130, 1900, p. 316. 